\title{
Controle e utilização da talidomida: adequados à ampliação do uso e mitigação do risco de teratogenicidade no Brasil?
}

\author{
Thalidomide control and use: are these appropriate to extend \\ the use and mitigate the risk of teratogenicity in Brazil?
}

Fernanda Torres Campos (https://orcid.org/0000-0002-2830-8221) 1,2

Roberta Márcia Marques dos Santos (https://orcid.org/0000-0003-1844-6628) ${ }^{2}$

Josilene Pereira Costa (https://orcid.org/0000-0002-8487-7066) ${ }^{3}$

Cristiane Aparecida Menezes de Pádua (https://orcid.org/0000-0001-7083-3188) ${ }^{1}$

${ }^{1}$ Universidade Federal de Minas Gerais. Av. Pres. Antônio Carlos 6627, Pampulha. 31270-901 Belo Horizonte MG Brasil.

fernanda_tcampos@ yahoo.com.br

${ }^{2}$ Fundação Ezequiel Dias.

Belo Horizonte MG Brasil.

${ }^{3}$ Secretaria Estadual de

Saúde de Minas Gerais. Belo

Horizonte MG Brasil.

\begin{abstract}
Drug utilization research to describe the control of thalidomide in Brazil and its use in Minas Gerais state. An online questionnaire was sent to the Brazilian federative units to collect data concerning distribution, dispensation, user registration, and thalidomide adverse events. Distribution (2011-2018) and dispensing (2015-2018) data in Minas Gerais were obtained through the pharmaceutical care management system. Analysis of variance and Tukey test were used for data comparisons. Of the 16 participating federative units, $100 \%$ and $50 \%$ used electronic distribution and dispensing systems, respectively, and about $43 \%$ registered users. Adverse event reporting systems were scarce. A $44 \%$ reduction was observed in the distribution in Minas Gerais for the period. Dispensing remained constant (mean 0.0004 DDD/1,000 inhabitants/day) and occurred mainly for erythema nodosum leprosum and multiple myeloma. Off-label use (2.2\%) was increasing. Most users were male (mean age 56 years) and thirty percent of women were of childbearing age. Thalidomide surveillance is a public health challenge. Despite the increased use and mandatory control, there is no national standardization, and adverse event reporting is incipient.

Key words Thalidomide, Teratogens, Drug Utilization, Pharmacovigilance
\end{abstract}

Resumo Estudo de utilização de medicamento que descreve o controle da talidomida no Brasil e sua utilização em Minas Gerais. Questionário online foi enviado às unidades federativas do Brasil para coleta de dados de distribuição, dispensação, cadastro de usuários e eventos adversos da talidomida. Dados de distribuição (2011-2018) e dispensação (2015-2018) de Minas Gerais foram obtidos pelo sistema de gerenciamento da assistência farmacêutica. Análise de variância e teste de Tukey foram empregados para comparações dos dados. Das 16 unidades federativas participantes, $100 \%$ e $50 \%$ utilizavam sistema eletrônico para distribuição e dispensação, respectivamente, e cerca de $43 \%$ cadastravam usuários. Sistemas de notificação de eventos adversos eram escassos. Houve redução de $44 \%$ na distribuição em Minas Gerais no período. A dispensação manteve-se constante (média 0,0004 DDD/1000 hab./dia) e ocorreu principalmente para eritema nodoso hansênico e mieloma múltiplo. O uso off-label (2,2\%) foi crescente. A maioria dos usuários era do sexo masculino (idade média 56 anos). Das mulheres, $30 \%$ estavam em idade fértil. A vigilância da talidomida é um desafio em saúde pública. Apesar da ampliação do uso e obrigatoriedade de controle, não há padronização em nível nacional e a notifcação de eventos adversos é incipiente.

Palavras-chave Talidomida, Teratogênese, Uso de Medicamentos, Farmacovigilância 


\section{Introdução}

A talidomida é um derivado do ácido glutâmico com propriedades analgésica, anti-inflamatória, imunomoduladora e antiangiogênica. Foi descoberta na Alemanha, em 1953, e utilizada principalmente para alívio de enjoos em gestantes ${ }^{1}$. A exposição in utero à talidomida levou ao nascimento de bebês com deformidades graves, resultando na retirada do mercado mundial em $1961^{2}$ e, em 1965, no Brasil ${ }^{3}$.

Em 1965, o médico Jacob Sheskin observou rápida melhora clínica das lesões de pacientes com eritema nodoso hansênico (ENH) após o uso de talidomida ${ }^{4}$. Ensaio clínico duplo-cego realizado pela Organização Mundial de Saúde (OMS), em 1971, confirmou sua eficácia no $\mathrm{ENH}^{5}$, e estudos subsequentes mostraram resultados promissores do uso da talidomida para tratamento de doenças infecciosas, autoimunes e cânceres ${ }^{6}$. No Brasil, seu uso é regulamentado pela RDC 11/2011 da Agência Nacional de Vigilância Sanitária (Anvisa) para tratamento de $\mathrm{ENH}$, úlceras aftoides idiopáticas em pessoas vivendo com HIV, lúpus, doença enxerto contra hospedeiro $(\mathrm{DECH})$, mieloma múltiplo $(\mathrm{MM})$ e síndrome mielodisplásica (SMD) $)^{7}$. Outras indicações da talidomida estão excepcionalmente previstas na Resolução como uso off-label e sua utilização tem sido observada no país ${ }^{8}$.

O desastre da talidomida nos anos 1950 e a ampliação do uso resultaram na adoção de medidas rígidas de controle do seu acesso em alguns países'. Em 1998, o Food and Drug Administration (FDA) autorizou a comercialização da talidomida nos Estados Unidos (EUA), exigindo o desenvolvimento de programas para monitorização do uso ${ }^{10}$. Para controlar a prescrição, dispensação e uso, o fabricante instituiu o programa Risk Evaluation and Mitigation Strategy - REMS ${ }^{\circledR 9-12}$ (inicialmente System for Thalidomide Education and Prescribing Safety - STEPS ${ }^{\circledR 9,10}$ ), baseado nos programas da isotretinoína e da clozapina9.

No Brasil, a talidomida é utilizada principalmente para tratamento do $\mathrm{ENH}$ e $\mathrm{MM}^{13}$. O país ocupa o segundo lugar no ranking mundial em incidência de hanseníase (13\%), sendo que $67,7 \%$ dos novos casos podem evoluir para $\mathrm{ENH}^{14}$. Toda cadeia de produção, distribuição, dispensação e notificação de eventos adversos da talidomida está sob gestão do Sistema Único de Saúde (SUS), e é regulamentada pela RDC 11/2011 que determina, entre outros, controle dos centros dispensadores e da prescrição, além do cadastro de usuários?
A notificação de eventos adversos da talidomida é compulsória ${ }^{7}$ e deve ser realizada pelo VigiMed e/ou pelo serviço de atendimento ao cliente do fabricante. Todavia, a farmacovigilância e o controle parecem não estar ocorrendo adequadamente. Estudo realizado em 2016 com pacientes em tratamento do ENH em hospital da rede sentinela de Minas Gerais observou diferenças importantes entre frequências do registro em prontuário e das notificações (compulsórias) à Anvisa e/ou ao fabricante de trombose e neuropatia associados a talidomida ${ }^{15}$. Ademais, casos de malformações fetais com fenótipo de embriopatia por talidomida ocorreram após $1965^{16-22}$.

O planejamento e a distribuição da talidomida às unidades federativas são atribuições do Ministério da Saúde. No entanto, há carência de estudos que descrevam os sistemas de controle de talidomida e como são efetivamente realizados a distribuição, dispensação e cadastro dos usuários. Além disso, não há dados divulgados, na literatura científica e em relatórios oficiais, quanto ao número de usuários do medicamento por indicação terapêutica no país.

O objetivo do estudo foi identificar e descrever os sistemas de controle da talidomida nas unidades federativas brasileiras e caracterizar a utilização em Minas Gerais, tendo em vista seu potencial teratogênico.

\section{Métodos}

\section{Desenho do estudo}

Estudo descritivo de utilização de medicamento para avaliar os sistemas de controle da talidomida no Brasil e a utilização em Minas Gerais, composto por: i) avaliação qualitativa da distribuição, dispensação, organização do cadastro de usuários e notificação de eventos adversos nas 27 unidades federativas e ii) avaliação qualitativa e quantitativa dos dados de distribuição, prescrição e dispensação em Minas Gerais.

O estudo foi aprovado pelo Comitê de Ética em Pesquisa da Universidade Federal de Minas Gerais (COEP/UFMG).

\section{Coleta de dados e variáveis}

\section{Gerenciamento e controle no Brasil}

A coleta de dados foi realizada por meio de questionário online (Google Forms), baseado na RDC 11/2011 e no sistema de gerenciamento da assistência farmacêutica de Minas Gerais. O 
questionário continha perguntas abertas e fechadas sobre cadastro de usuários, controle da distribuição e dispensação (uso de sistemas eletrônicos nacional ou próprios, conteúdo e transmissão de dados) e sistema para notificação de eventos adversos (e.g. conteúdo, acompanhamento e envio de dados a entes do SUS).

$\mathrm{O}$ estudo piloto foi realizado enviando o questionário à Assistência Farmacêutica da Secretaria Estadual de Saúde (SES) de Minas Gerais para testar a adequação do instrumento e procedimentos da coleta de dados. Contatos telefônicos identificaram os responsáveis pela Assistência Farmacêutica de todas as SES, prosseguindose com encaminhamento do questionário por e-mail. Foram realizadas até seis tentativas de contato telefônico e envio do questionário por até três vezes.

\section{Utilização em Minas Gerais}

Dados de distribuição e dispensação da talidomida do sistema de gerenciamento da assistência farmacêutica foram disponibilizados pela SES de Minas Gerais. Dados de distribuição, representativos de todas as unidades dispensadoras do estado, de janeiro de 2011 a novembro de 2018 , contemplavam data da solicitação do medicamento, nome da regional de saúde e quantidade de comprimidos. Dados de dispensação foram analisados de janeiro de 2015 a dezembro de 2018 contendo data da dispensação; nome, regional e município da unidade de saúde; código de identificação, data de nascimento, sexo e município de residência do paciente; Classificação Internacional de Doenças (CID-10)/indicação terapêutica; conselho regional e especialidade do prescritor, e quantidade dispensada. Uso off-label foi caracterizado pela dispensação da talidomida para indicações terapêuticas diferentes daquelas aprovadas pela RDC 11/2011 (ENH, úlceras aftoides idiopáticas, lúpus, DECH, MM e SMD).

Dados de distribuição e de dispensação foram expressos em Dose Diária Definida (DDD) ${ }^{23}$ e em DDD/1.000 hab./dia, calculada pela fórmula: $\mathrm{DDD} / 1.000 \mathrm{hab} . / \mathrm{dia}=\mathrm{CMA}(\mathrm{mg})^{\star} 1.000 / \mathrm{DD}-$ $\mathrm{D}^{\star}$ pop. ${ }^{\star} 365$ dias, com $\mathrm{CMA}=$ quantidade média (miligramas) de talidomida no período; DD$\mathrm{D}=$ dose diária definida da talidomida $(100 \mathrm{mg})$ na principal indicação em adultos $(\mathrm{ENH})^{23}$; pop.=população de Minas Gerais estimada pelo IBGE para 2015 (20.648.978 habitantes).

\section{Análise de dados}

Análises descritivas foram realizadas para caracterização das variáveis por meio de frequências absolutas e relativas. Média, desvio-padrão e intervalo de confiança (IC) de 95\% foram calculados para dados de distribuição e dispensação. A análise de variância (ANOVA) foi empregada para comparar diferenças entre as médias da dispensação por usuários entre 2015 e 2018 e múltiplas comparações foram baseadas no teste de Tukey, considerando nível de significância de 0,05 . O manejo e análises de dados foram realizados pelo Statistical Analysis System (SAS), versão 9.4 .

\section{Resultados}

\section{Gerenciamento e controle no Brasil}

Dezesseis (59\%) unidades federativas responderam ao questionário (Tabela 1 ). As regiões Sul e Sudeste obtiveram 100\% de participação, enquanto Norte, Centro-Oeste e Nordeste tiveram representação de 28,6\% $(n=2), 50 \%(n=2)$ e $55,6 \%(n=5)$, respectivamente.

Nove $(56,3 \%)$ secretarias não realizam cadastro de usuários ou não informaram. Todas relataram utilizar sistemas eletrônicos (Sistema Nacional de Gestão da Assistência Farmacêutica [Hórus] e/ou sistema próprio) para a distribuição. Oito secretarias (50\%) não possuem sistemas de controle para dispensação e utilizam planilhas Excel e livros de controle $(n=3)$, ou o sistema é desconhecido ou inexistente $(n=5)$. A outra metade informou utilizar sistemas eletrônicos para controlar a dispensação (Hórus [n=2], sistemas próprios [ $n=5]$, Hórus juntamente com sistema próprio e dispensação manual [n=1]).

Apenas uma secretaria possui sistema próprio de notificação, que fornece dados do paciente (nome, data de nascimento, sexo, endereço, CID, dados clínicos e evolução do quadro, fármacos concomitantes e doenças), do medicamento (nome, número do lote) e dos eventos adversos (evento, gravidade, data da ocorrência), sendo possível acompanhar sua evolução, enviar à Anvisa e a outros entes do SUS. As unidades que não possuem sistemas de envio de notificação $(n=14)$ informaram encaminhá-la a uma ou mais instituições como Anvisa, Ministério da Saúde ou fabricante. 


\section{Utilização em Minas Gerais}

\section{Distribuição e dispensação}

Entre janeiro de 2011 a novembro de 2018, foram distribuídos 3.493.230 comprimidos de talidomida, equivalente a 3.493.230 DDD ou à média de 0,0006 DDD/1.000 hab./dia (Tabela 2). Houve uma redução de $44,3 \%$ na distribuição no período. Quatro das 28 regionais de saúde foram responsáveis por aproximadamente 50\%

Tabela 1. Utilização de sistemas de cadastro de usuários de talidomida, controle da distribuição e dispensação, e do sistema para notificação de eventos adversos nas unidades federativas brasileiras $(n=16)$.

\begin{tabular}{|c|c|}
\hline Características $(n=16)$ & n $(\%)$ \\
\hline \multicolumn{2}{|l|}{ Cadastro de usuários } \\
\hline Realiza cadastro & $7(43,8)$ \\
\hline Não realiza cadastro & $1(6,3)$ \\
\hline Não informado & $8(50,0)$ \\
\hline \multicolumn{2}{|l|}{ Sistema para cadastro } \\
\hline Hórus & $1(6,3)$ \\
\hline Hórus e sistema próprio & $1(6,3)$ \\
\hline Hórus e Excel & $1(6,3)$ \\
\hline Excel & $2(12,5)$ \\
\hline Sistema próprio & $1(6,3)$ \\
\hline Sistema próprio e Excel & $1(6,3)$ \\
\hline Inexistente & $1(6,3)$ \\
\hline Não se aplica & $8(50,0)$ \\
\hline Encaminha os dados ao Ministério da Saúde & $6(37,5)$ \\
\hline Não encaminha os dados ao Ministério da Saúde & $1(6,3)$ \\
\hline Processo de encaminhamento dos dados ao Ministério da Saúde desconhecido & $1(6,3)$ \\
\hline Não se aplica & $8(50,0)$ \\
\hline \multicolumn{2}{|l|}{ Distribuição } \\
\hline Existência de sistema de controle ( $\operatorname{sim}$ ) & $16(100,0)$ \\
\hline Ausência de sistema de controle (não) & $0(0,0)$ \\
\hline \multicolumn{2}{|l|}{ Sistema de distribuição } \\
\hline Hórus & $7(43,8)$ \\
\hline Hórus e sistema próprio & $1(6,3)$ \\
\hline Sistema próprio & $8(50,0)$ \\
\hline Encaminha os dados ao Ministério da Saúde & $4(25,0)$ \\
\hline \multicolumn{2}{|l|}{ Dispensação $(n=16)$} \\
\hline Existência de sistema de controle (sim) & $8(50,0)$ \\
\hline Ausência de sistema de controle (não) & $8(50,0)$ \\
\hline \multicolumn{2}{|l|}{ Sistema de dispensação } \\
\hline Hórus & $2(12,5)$ \\
\hline Hórus, sistema próprio e manual & $1(6,3)$ \\
\hline Sistema próprio & $5(31,3)$ \\
\hline Livros de controle e planilha Excel & $3(18,8)$ \\
\hline Desconhecido ou inexistente & $5(31,3)$ \\
\hline Encaminha os dados ao Ministério da Saúde & $7(43,8)$ \\
\hline \multicolumn{2}{|l|}{ Notificação de evento adverso $(n=16)$} \\
\hline Existência de sistema de notificação (sim) & $1(6,3)$ \\
\hline Ausência de sistema de notificação (não) & $14(87,5)$ \\
\hline Processo de notificação desconhecido & $1(6,3)$ \\
\hline Encaminha os eventos adversos a entes do SUS & $15(93,8)$ \\
\hline Anvisa & $10(66,7)$ \\
\hline Ministério da Saúde & $8(53,3)$ \\
\hline Fabricante & $3(20,0)$ \\
\hline Outros entes do SUS & $3(20,0)$ \\
\hline
\end{tabular}

Fonte: Elaborada pelas autoras. 
da distribuição (Belo Horizonte 31,4\%; Uberlândia 8,0\%; Coronel Fabriciano 6,0\%; e Uberaba $5,6 \%)$. As demais representaram, individualmente, $4,0 \%$ ou menos do total distribuído no estado.

A dispensação manteve-se relativamente constante a partir do ano de 2016 (valor-p>0,05) . No período 2015-2018, representou $83,0 \%$ da quantidade distribuída, correspondendo à média de 0,0004 DDD/1000 hab./dia.

\section{Usuários}

A maioria era do sexo masculino, idade igual ou superior a 50 anos (média 56 anos), registro de um diagnóstico clínico e residia em Belo Horizonte. Um total de 506 mulheres (10\% dos participantes e $30 \%$ das mulheres) estava em idade fértil (entre 10 e 49 anos) (Tabela 3).

\section{Diagnósticos}

Do total de dispensações $(n=29.212)$ no período de quatro anos, a maior parte foi para o tratamento do ENH (54,5\%), MM (27,3\%), lúpus $(6,0 \%)$ e usos off-label (2,2\%). Dispensações para úlceras aftoides, SMD e DECH corresponderam, juntas, a $<1 \%$ dos registros. Houve aumento do número de dispensações para MM (127,9\%), seguida por lúpus $(81,1 \%)$, usos off-label $(76,3 \%)$, ENH $(34,7 \%)$ e SMD (15,8\%). Observou-se melhora no registro dos diagnósticos, notando-se redução de 99\% do número de dados ignorados (Figura 1).

\section{Uso off-label}

Foram verificados 642 registros de dispensação de talidomida para 39 usos off-label distintos.

Tabela 3. Características dos usuários de talidomida atendidos nas unidades dispensadoras de Minas Gerais, 2015-2018 ( $\mathrm{n}=4.625)$.

\begin{tabular}{lr}
\hline \multicolumn{1}{c}{ Características } & \multicolumn{1}{c}{$\mathbf{n}(\%)$} \\
\hline Sexo & $2.802(60,6)$ \\
Masculino & $1.823(39,4)$ \\
Feminino & $506(10,9)$ \\
$\quad$ Idade fértil ${ }^{\star}$ & \\
Idade (anos) & $13(0,3)$ \\
$\leq 9$ & $1.474(31,9)$ \\
$10-49$ & $2.941(63,6)$ \\
$\geq 50$ & $197(4,3)$ \\
Desconhecido & \\
No de diagnósticos & $3.346(72,3)$ \\
1 & $1.279(27,7)$ \\
2 ou mais & \\
Município de residência & $423(9,1)$ \\
Belo Horizonte & $254(5,5)$ \\
Uberlândia & $138(3,0)$ \\
Uberaba & $108(2,3)$ \\
Contagem & $103(2,2)$ \\
Montes Claros & $3.599(77,8)$ \\
Outros (n=579) &
\end{tabular}

${ }^{*}$ Idade fértil: 10 a 49 anos.

Fonte: Sistema de Gerenciamento da Assistência Farmacêutica de Minas Gerais (SIGAF).

Tabela 2. Distribuição e dispensação de talidomida em Minas Gerais, 2011-2018.

\begin{tabular}{lcccc}
\hline \multirow{2}{*}{ Período } & \multicolumn{2}{c}{ Distribuição } & Dispensação & DDD/usuário \\
\cline { 2 - 4 } & DDD & Registros (n) & DDD & --- \\
\hline 2011 & 582.090 & --- & --- & --- \\
2012 & 433.410 & --- & --- & --- \\
2013 & 468.540 & --- & --- & --- \\
2014 & 462.120 & --- & -- & 171,0 \\
2015 & 403.050 & 7.402 & 333.722 & 329,7 \\
2016 & 327.090 & 6.721 & 298.369 & 358,0 \\
2017 & 492.450 & 7.421 & 330.424 & 379,7 \\
2018 & $324.480^{*}$ & 7.668 & $320.879^{*}$ & --- \\
Média & 436.654 & 7.303 & 320.849 & \\
(DP) & $(85.905)$ & $(406,5)$ & $(15.945)$ & --- \\
IC95\% & $364.836-508.472$ & $230,3-1516$ & $295.476-346.221$ &
\end{tabular}

$\mathrm{DDD}=$ Dose diária definida (1 DDD talidomida=100mg); DP=desvio-padrão; IC95\%=intervalo de confiança 95\%.

${ }^{\star}$ Valor-p<0,0001 - Anova/Teste de Tukey (a diferença observada entre as médias é devida ao ano de 2015 em relação à 2016,2017 e 2018). \#Janeiro a novembro de 2018. 


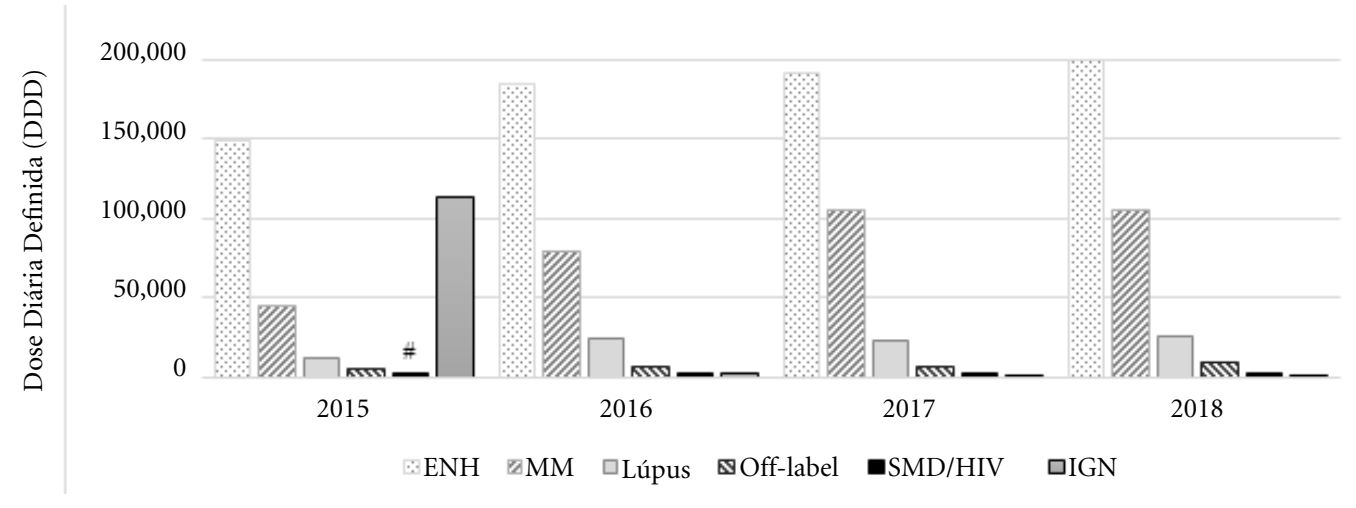

Figura 1. Dispensação da talidomida (em DDD) por indicação do medicamento em Minas Gerais, 2015-2018. \#Corresponde às indicações SMD/HIV/DECH. IGN=Indicação Clínica Ignorada; DDD (100 mg) é calculada com base na principal indicação de uso da talidomida (ENH).

Fonte: Sistema de Gerenciamento da Assistência Farmacêutica de Minas Gerais (SIGAF).

Doença de Behçet $(n=113)$, estomatite $(n=83)$, líquen $(n=79)$, prurigo nodular $(n=75)$ e doenças pulmonares $(n=71)$ representaram mais da metade dos registros.

\section{Especialidade do prescritor}

Informação sobre especialidade médica não foi preenchida em 83,0\% ( $n=24.229)$ dos registros de dispensação. Considerando os registros preenchidos, a talidomida foi prescrita por clínicos $(61,5 \%)$, dermatologistas $(18,4 \%)$ e hematologistas $(10,3 \%)$. As demais $(9,8 \%)$ foram de outras 14 especialidades médicas.

\section{Discussão}

O estudo avaliou a estrutura de controle da talidomida no Brasil e a utilização em Minas Gerais. A ampliação das indicações de uso da talidomida no país e, em menor extensão no mundo, impõe a necessidade de vigilância e controle efetivos. A suspeita de casos de malformações congênitas associadas ao seu uso, após 1965, suscita fragilidades no controle 16-22 $^{\text {. }}$

$\mathrm{Na}$ análise diagnóstica do controle da talidomida obteve-se aproximadamente $60 \%$ de participação das unidades federativas. Nas regiões Norte, Centro-Oeste e Nordeste, onde taxas de detecção de hanseníase são maiores ${ }^{24}$, a representatividade foi menor. Tal fato sugere iniquidades na infraestrutura de controle e gerenciamento da talidomida, podendo refletir dificuldades no monitoramento do uso. Registros de vítimas da síndrome de talidomida da $3^{\mathrm{a}}$ (2005-2010), 4a e $5^{\mathrm{a}}$ gerações (após 2010) no Brasile ${ }^{22}$, concentraram-se na região Nordeste. A não resposta de algumas unidades federativas dessa região, pode estar relacionada à falta de infraestrutura ou ao receio de explicitar possíveis fragilidades dos processos de controle. Em contraste, as regiões Sudeste e Sul com mais alto desenvolvimento industrial e urbano $(58,2 \%$ e $14,2 \%$ do Produto Interno Bruto do país, respectivamente) tiveram $100 \%$ de participação. Supõe-se que essas regiões possuam estrutura organizacional que permita melhor gerenciamento dos processos, podendo resultar em melhor controle, porém sem refletir a realidade nacional.

O Ministério da Saúde é responsável pela criação e manutenção do cadastro nacional de usuários de talidomida, que deve ser realizado pela assistência farmacêutica das unidades federativas $^{7}$. Apesar da maioria (6/8) das secretarias relatar que a relação de usuários cadastrados é repassada ao Ministério da Saúde, dados sobre número e perfil de usuários não estão disponíveis em relatórios oficiais publicados e não podem ser acessados na literatura devido à escassez de estudos nacionais sobre a utilização desse medicamento.

O foco do gerenciamento e do controle pa- 
rece se concentrar no planejamento e na distribuição da talidomida, considerando a utilização mais frequente de algum meio de controle para essa atividade comparada à dispensação. Isso demonstra que o uso de sistemas informatizados para o registro da dispensação de medicamentos ainda não é uma realidade no Brasil ${ }^{25}$. Apesar da disponibilidade do Hórus como sistema de gestão da assistência farmacêutica no país, observou-se sua baixa utilização para gerenciamento da distribuição e, principalmente, da dispensação de talidomida. Esse sistema contém informações sobre controle de estoque, dados de dispensação, do paciente, do prescritor e da indicação de uso $^{26}$. Apesar disso, muitas unidades federativas parecem optar por desenvolver e utilizar sistemas próprios para gerenciar seus dados.

É importante que sistemas informatizados nacionais ou próprios das secretarias sejam utilizados e que haja um fluxo de informação para construção de bases de dados de abrangência nacional que subsidiem ações de farmacovigilância, pesquisas e tomadas de decisões em saúde ${ }^{25}$. O Sistema de Controle Logístico de Medicamentos (SICLOM) e o Sistema Nacional de Gerenciamento de Produtos Controlados (SNGPC) são exemplos de bases de dados sobre medicamentos empregadas para condução de estudos de utilização e auxílio no monitoramento do uso de medicamentos no Brasil ${ }^{27}$. Registros sobre o consumo da talidomida, contendo características do usuário, como sexo e data de nascimento, podem direcionar a farmacovigilância para grupos prioritários (e.g. pacientes com lúpus, predominantemente mulheres em idade fértil, e pacientes idosos com MM em risco de desenvolver neuropatia e eventos tromboembólicos $)^{13}$, além de facilitar a realização de pesquisas.

Eventos adversos e queixas técnicas da talidomida devem ser compulsoriamente notificados à Anvisa pelo VigiMed ${ }^{7}$. Embora grande parte das secretarias tenha declarado notificar eventos adversos, estudo recente mostrou subnotificação de eventos adversos da talidomida à Anvisa (15 notificações em seis anos) em comparação aos relatos espontâneos ao laboratório fabricante $(n=23)$ e principalmente aos registros de eventos adversos $(n=1.356)$ em prontuários de pacientes com ENH no período de um ano de tratamento ${ }^{15}$. É fundamental que os formulários de notificação sejam simples, acessíveis e os profissionais sejam constantemente treinados para identificação e notificação de eventos adversos ${ }^{28}$. Com a substituição do Notivisa pelo VigiMed, utilizado pelo centro colaborador da OMS para monitoramen- to internacional de medicamentos, espera-se melhorar o fluxo de informações com geração de relatórios mais fidedignos sobre eventos adversos.

O programa de controle do uso da talidomida nos EUA, $\mathrm{REMS}^{\circledR}$, foi criado e aprimorado para evitar casos de teratogenicidade e é considerado efetivo $^{10-12}$. A RDC 11/2011 foi estabelecida com base no REMS $^{\circledR}$ e apresenta medidas de controle similares, tais como limitação do acesso apenas a farmácias, prescritores e pacientes elegíveis registrados; instrução e aconselhamento dos pacientes; preenchimento de consentimento informado; realização de testes de gravidez; e etiquetas de alertas sobre teratogenicidade na embalagem do medicamento ${ }^{7}$. No entanto, não se podem considerar efetivos o controle e a utilização da talidomida, uma vez que não há consolidação do cadastro de usuários, sistemas adequados e em pleno funcionamento para dispensação, distribuição e notificação de eventos adversos.

O controle da distribuição da talidomida em Minas Gerais é realizado exclusivamente pelo sistema de gerenciamento da assistência farmacêutica, diferentemente da dispensação. A maior distribuição da talidomida no período (20112018) ocorreu em três dos quatro municípios mais populosos do estado e com maior número de casos de hanseníase. A redução da distribuição, principalmente de 2011 para 2012 (25,5\%), pode refletir melhora na utilização do sistema, considerando que foi inicialmente implantado para gerenciar recursos e, na sequência, para efetivo controle. Consequentemente, de 2012 a 2014, a distribuição permaneceu relativamente constante. A redução da quantidade distribuída e dispensada, em 2016, pode ser atribuída ao desabastecimento do medicamento em 2015, que refletiu no ano seguinte. Em 2017, houve crescimento exacerbado da distribuição, sem alteração significativa da dispensação em relação aos anos anteriores. Pode-se inferir que o crescimento se deva ao hábito de aumentar o estoque após situações atípicas de falta de medicamentos, já que, em 2018, a distribuição e a dispensação se normalizaram.

O perfil de usuários de talidomida corrobora os achados de outros estudos realizados no Brasil, mostrando predominância do sexo masculino, idade superior a 45 anos e diagnóstico de $\mathrm{ENH}^{13,29}$. O estudo identificou percentual considerável de dispensações para mulheres em idade fértil, reforçando a importância do controle rigoroso da dispensação com realização de teste de gravidez, orientação para utilização de método contraceptivo e acompanhamento ${ }^{7}$. Além 
das características do usuário, o preenchimento completo do diagnóstico nas bases de dados fornece informações relevantes sobre seu perfil, indicando pacientes em situação de fragilidade social (com $\mathrm{ENH}^{15}$ ), predominantemente idosos (em tratamento do MM) e mulheres em idade fértil com lúpus.

Abordagens educacionais e farmacovigilância são fundamentais para prevenção da teratogenicidade como os casos incidentes após 1965, atribuídos ao controle deficiente do medicamento ${ }^{16-22,30}$. Soma-se a isso a baixa escolaridade dos pacientes com $\mathrm{ENH}^{29}$, aumentando o risco de erros de medicação e eventos adversos por dificuldade na compreensão dos cuidados necessários para o tratamento. A utilização de linguagem adequada para orientação ao paciente e seu acompanhamento são medidas de farmacovigilância que podem mitigar o risco de eventos adversos graves como malformações congênitas, eventos tromboembólicos e neuropatia.

A diminuição da dispensação da talidomida (2015-2018) está em concordância com outro estudo que mostrou redução do número de prescrições em 2011 comparado com $2001^{13}$. A promulgação da RDC 11/2011 e a aprovação de protocolos clínicos, em 2011, podem explicar esses resultados. No presente estudo, a melhora do preenchimento dos campos do sistema de gerenciamento da assistência farmacêutica foi o que mais influenciou a qualidade dos dados.

Apesar do aumento da dispensação ter sido maior para o MM, o ENH continua representando a principal indicação da talidomida ${ }^{13,31}$. A hanseníase é uma doença de notificação compulsória no Brasil, segundo país do mundo em incidência da doença (13\%), perdendo apenas para Índia $(60 \%)^{14}$. Em Minas Gerais, a notificação de hanseníase permaneceu constante entre 2015$2018^{24}$. Comparativamente, há poucos dados epidemiológicos disponíveis para o MM, câncer raro e incurável que acomete principalmente idosos ${ }^{32}$; portanto, uma doença em ascensão, devido ao envelhecimento populacional. A talidomida compõe o esquema de primeira escolha para o tratamento do MM no SUS, sua segunda principal indicação $0^{13}$. É segunda escolha para as demais indicações, que são pouco frequentes e possuem esquemas posológicos diversos, justifica o aumento discreto do número de dispensações, como por exemplo para a SMD $(<1 \%)$ incluída em 2015.

No Brasil, não se conhece o número de usuários de talidomida, em parte porque apenas a hanseníase é de notificação compulsória, o me- dicamento é primeira escolha para duas das seis indicações (ENH e MM), e também pela indisponibilidade de dados oficiais.

A dispensação para uso off-label da talidomida é permitida pela Anvisa nas situações consideradas indispensáveis e como última alternativa terapêutica ${ }^{7}$. Observou-se aumento do uso off-label, embora ainda represente pequena proporção em relação a todas as dispensações, como já observado previamente ${ }^{13}$. Esse resultado deve ser representado com cautela, uma vez que esse aumento pode ser devido à melhora na qualidade do preenchimento dos diagnósticos. Por outro lado, é muito provável que esse uso esteja subnotificado em decorrência de exigências de justificativa do uso excepcional pelo prescritor, o que dificulta o controle e o avanço na construção de evidências clínicas de segurança e eficácia. Estudos apontam para a necessidade de pesquisas sobre o uso off-label considerando o mercado farmacêutico, relações jurídicas, segurança e eficácia dos produtos e atuação das agências reguladoras para racionalizar o processo decisório e aperfeiçoar o controle ${ }^{33}$.

Nas dispensações, $83,0 \% \quad(n=24.229)$ dos campos "Especialidade do Prescritor" não estavam preenchidos. Nos registros válidos, verificouse conformidade da maior parte das especialidades médicas registradas com os usos clínicos da talidomida, como já relatado em outro estudo ${ }^{13}$.

A avaliação qualitativa mostrou que não há padronização do uso de sistemas de controle e gerenciamento da talidomida. A ausência de resposta das regiões Centro-Oeste, Norte e Nordeste limitam nossos achados, mas o levantamento apresentado pelo estudo, aliado à ocorrência tardia de casos de malformação congênita no Brasi $^{22}$, indicam fragilidades importantes no controle do medicamento em contraposição à ampliação de seu uso no país. Estudos adicionais a esse primeiro diagnóstico devem ser realizados para avaliar de forma contínua as necessidades regionais dos sistemas de controle e gerenciamento da talidomida.

A incompletude de dados sobre a utilização da talidomida em Minas Gerais (perda quantitativa de dados da dispensação e de informações relativas à doença, características dos usuários $\mathrm{e}$ do prescritor) e o período relativamente curto disponível para análise, compreendem limitações dessa avaliação. Contudo, as alterações observadas na dispensação da talidomida estão alinhadas à diminuição do uso no ENH e aumento de uso no $\mathrm{MM}^{13}$. $\mathrm{O}$ aperfeiçoamento do sistema de gerenciamento (e.g. redução da proporção de dados ignorados) deverá contribuir para estudos farma- 
coepidemiológicos futuros. A disponibilidade de bases de dados secundários permite acesso a informações rotineiramente coletadas para condução de pesquisas sobre utilização de medicamentos direcionando tomadas de decisão em saúde.

A vigilância da talidomida é um grande desafio em saúde pública, não obstante toda história trágica. Logo, é necessário fiscalização do funcionamento da estrutura existente para mitigar

\section{Colaboradores}

FT Campos: concepção, planejamento, análise, interpretação e redação do trabalho. RMM Santos: concepção, planejamento, análise, interpretação e redação do trabalho. JP Costa: análise e interpretação dos resultados do trabalho. CAM Pádua: concepção, planejamento, análise, interpretação e redação do trabalho.

\section{Agradecimentos}

Os autores agradecem às Secretarias de Estado de Saúde pela disponibilidade em contribuir com o estudo. Nosso agradecimento também à pesquisadora Raíssa Carolina Fonseca Cândido pelo auxílio na construção do questionário online. eventos adversos, sobretudo a teratogenicidade, $\mathrm{e}$ melhorar a qualidade do cuidado. A ampliação do uso off-label da talidomida demonstra que, apesar de ser a substância que provocou um dos maiores desastres do século XX, se figura como medicamento promissor no século $\mathrm{XXI}^{34}$, e reforça a necessidade de maior rigor no monitoramento da utilização.

\section{Referências}

1. Tseng S, Pak G, Washenik K, Pomeranz MK, Shupack JL. Rediscovering thalidomide: a review of its mechanism of action, side effects, and potential uses. J Am Acad Dermatol 1996; 35(6):969-979.

2. McBride WG. Thalidomide and Congenital Abnormalities. Lancet 1961; 278(7216):1358.

3. Associação Brasileira dos Portadores da Síndrome da Talidomida. Talidomida, O que é Talidomida? [Internet]. 2019 [acessado 2019 jan 20]. Disponível em: http://www.talidomida.org.br/oque.asp.

4. Sheskin J. Thalidomide in the treatment of lepra reactions. J Clin Pharm Ther 1965; 6:303-306.

5. Iyer CG, Languillon J, Ramanujam K, Tarabini-Castellani G, De las Aguas JT, Bechelli LM, Uemura K, Martinez Dominguez V, Sundaresan T. WHO co-ordinated short-term double-blind trial with thalidomide in the treatment of acute lepra reactions in male lepromatous patients. Bull WHO 1971; 45(6):719-732.

6. Chen M, Doherty SD, Hsu S. Innovative uses of thalidomide. Dermatol Clin 2010; 28(3):577-586.

7. Brasil. Resolução RDC no 11, de 22 de março de 2011. Dispõe sobre o controle da substância talidomida e do medicamento que a contenha. Diário Oficial da União 2011; 24 mar.

8. Duarte BKL, Souza SM, Costa-Lima C, Medina SS, Ozelo MC. Thalidomide for the Treatment of Gastrointestinal Bleeding Due to Angiodysplasia in a Patient with Glanzmann's Thrombasthenia. Hematol Rep 2017; 9(2):6961.

9. Zeldis JB, Williams BA, Thomas SD, Elsayed ME. S.T.E.P.S.: a comprehensive program for controlling and monitoring access to thalidomide. Clin Ther 1999; 21(2):319-330.

10. Uhl K, Cox E, Rogan R, Zeldis JB, Hixon D, Furlong LA, Singer S, Holliman T, Beyer J, Woolever W. Thalidomide use in the US: experience with pregnancy testing in the S.T.E.P.S. programme. Drug Saf 2006; 29(4):321-329.

11. Public Affairs Committee. Teratology society public affairs committee position paper: thalidomide. Teratology 2000; 62(3):172-173. 
12. Brandenburg NA, Bwire R, Freeman J, Houn F, Sheehan P, Zeldis JB. Effectiveness of Risk Evaluation and Mitigation Strategies (REMS) for Lenalidomide and Thalidomide: Patient Comprehension and Knowledge Retention. Drug Saf 2017; 40(4):333-341.

13. Paumgartten FJ, Souza NR. Clinical use and control of the dispensing of thalidomide in Brasilia-Federal District, Brazil, from 2001 to 2012. Cien Saude Colet 2013; 18(11):3401-3408.

14. World Health Organization (WHO). Department of Control of Neglected Tropical Diseases. Leprosy elimination. Global leprosy update, 2015: time for action, accountability and inclusion. Wkly Epidemiol Rec 2015; 91(35):405-420.

15. Drummond PLM, Santos RMM, Silva CA, Pádua CAM. Pharmacovigilance of thalidomide in the Brazilian Healthy System and patient safety. Braz J Pharm Sci 2020; 56:e18726.

16. Castilla EE. Thalidomide, a current teratogen in South America. Teratology 1996; 54(6):273-277.

17. Paumgartten FJ, Chahoud I. Thalidomide embryopathy cases in Brazil after 1965. Reprod Toxicol 2006; 22(1):1-2.

18. Schuler-Faccini L, Soares RCF, Sousa ACM, Maximino C, Luna E, Schwartz IVD, Waldman C, Castilla EE. New cases of thalidomide embryopathy in Brazil. Birth Defects Res A Clin Mol Teratol 2007; 79(9):671-672.

19. Vianna FSL, Lopez-Camelo JS, Leite JCL, Sanseverino MTV, Dutra MG, Castilla EE, Schuler-Faccini L. Epidemiological surveillance of birth defects compatible with thalidomide embryopathy in Brazil. PLoS One 2011; 6(7):e21735.

20. Kowalski TW, Sanseverino MTV, Schuler-Faccini L, Vianna FSL. Thalidomide embryopathy: Follow-up of cases born between 1959 and 2010. Birth Defects Res A Clin Mol Teratol 2015; 103(9):794-803.

21. Vianna FSL, Oliveira MZ, Sanseverino MTV, Morelo EF, Rabello Neto DL, Lopez-Camelo J, Camey SA, Schuler-Faccini L. Pharmacoepidemiology and thalidomide embryopathy surveillance in Brazil. Reprod Toxicol 2015; 53:63-67.

22. Agência Nacional de Vigilância Sanitária (Anvisa). Gerência de Produtos Controlados. $16^{\circ}$ Encontro Nacional da Rede Sentinela [Internet]. 2019 [acessado 2020 maio 13]. Disponível em: http://portal.anvisa.gov.br/documents/33868/5240909/9-Gest\%C3\%A3o+da+prescri\%C3\%A7\%C3\%A3o\%2C+dispensa \%C3\%A7\%$\mathrm{C} 3 \% \mathrm{~A} 3 \mathrm{o}+\mathrm{e}+$ monitoramento+de+uso+do+medicamento+Talidomida__L\%C3\%9ACIA+SURITA. pdf/2aa95f3c-4713-4097-85c1-c149c8a46141

23. World Health Organization (WHO). WHO Collaborating Centre For Drug Statistics Methodology. Anatomical therapeutic chemical (ATC) classification index [Internet]. Oslo: WHO-Oslo; 2019 [acessado 2019 jun 10]. Disponível em: https://www.whocc.no/ atc_ddd_index/.

24. Brasil. Ministério da Saúde (MS). Banco de dados do Sistema Único de Saúde - DATASUS. Informações de Saúde [Internet]. 2019 [acessado 2019 jun 10]. Disponível em: http://tabnet.datasus.gov.br/cgi/tabcgi. exe?sinannet/hanseniase/cnv/hanswuf.def.
25. Barbosa MM, Garcia MM, Nascimento RCRM, Reis EA, Guerra-Júnior AA, Acúrcio FJ, Álvares J. Infrastructure evaluation of Pharmaceutical Services in the National Health System of Minas Gerais. Cien Saude Colet 2017; 22(8):2475-2486.

26. Curso para Qualificação de Profissionais da Assistência Farmacêutica e Capacitação para o Sistema Hórus. Relatórios Hórus. Manual do Sistema Hórus. Manual 6 [Internet]. 2019 [acessado 2019 maio 13]. Disponível em: http://portalarquivos2.saude.gov.br/images/ pdf/2015/setembro/14/Manual-6---Relat--rios-H-RUS.pdf.

27. Madruga LGSL, Silva GVV, Alves VAR, Azeredo TB, Setúbal S, Brito MA, Lima EC. Aspects related to the use of antiretrovirals in high complexity patients in the state of Rio de Janeiro. Cien Saude Colet 2018; 23(11):3649-3662.

28. Massele A, Burguer J, Kalemeera F, Jande M, Didimalang T, Kalungia AC, Matshotyana K, Law M, Malone B, Ogunleye O, Oluka M, Paramadhas BD, Rwegerera G, Zinyowera S, Godman B. Outcome of the second Medicines Utilisation Research in Africa Group meeting to promote sustainable and appropriate medicine use in Africa. Expert Rev Pharmacoecon Outcomes Res 2017; 17(2):149-152.

29. Drummond PLM, Santos RMM, Carvalho GO, Menezes de Pádua CA. Adverse events in patients with leprosy on treatment with thalidomide. Rev Soc Bras Med Trop 2019; 52:e20180385.

30. Oliveira MA, Bermudez JAZ, Souza ACM. Talidomida no Brasil: vigilância com responsabilidade compartilhada? Cad Saude Publica 1999; 15(1):99-112.

31. Costa PSS, Fraga LR, Kowalski TW, Daxbacher ELR, Schuler-Faccini L, Vianna FSL. Erythema Nodosum Leprosum: Update and challenges on the treatment of a neglected condition. Acta Trop 2018; 183:134-141.

32. Raab MS, Podar K, Breitkreutz I, Richardson PG, Anderson KC. Multiple myeloma. Lancet 2009; 374(9686):324-339.

33. Nobre PF. Off-label prescriptions in Brazil and in the US: legal aspects and paradoxes. Cien Saude Colet 2013; 18(3):847-854.

34. Hassan I, Dorjay K, Anwar P. Thalidomide in dermatology: revisited. Indian J Dermatol 2015; 60(2):213.

Artigo apresentado em 17/12/2019

Aprovado em 14/07/2020

Versão final apresentada em 16/07/2020

Editores-chefes: Romeu Gomes, Antônio Augusto Moura da Silva 